\title{
Structural Probability Concepts Adapted to Electrical Engineering
}

Eric P. Steinberg

Ohio University

Athens, Ohio

and

Christos C. Chamis

Lewis Research Center

Cleveland, Ohio

Prepared for the 35th AIAA Structures, Structural Dynamics and Materials Conference sponsored by the American Institute of Aeronautics and Astronautics Hilton Head, South Carolina, April 18-20, 1994

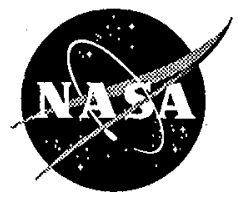

National Aeronautics and Space Administration

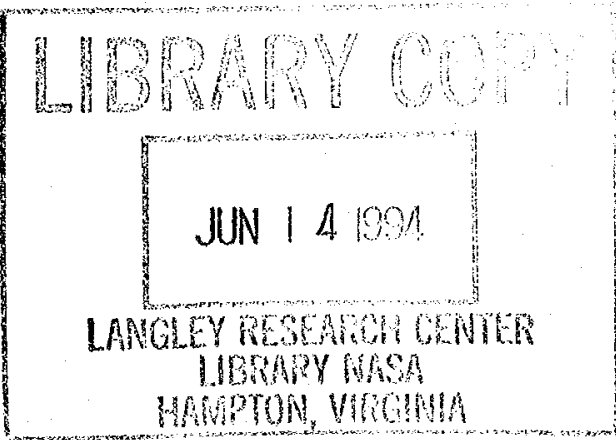


. 


\title{
STRUCTURAL PROBABILITY CONCEPTS ADAPTED TO ELECTRICAL ENGINEERING
}

\author{
Eric P. Steinberg \\ Department of Civil Engineering \\ Ohio University \\ Athens, Ohio 45701 \\ and \\ Christos C. Chamis \\ National Aeronautics and Space Administration \\ Lewis Research Center \\ Cleveland, Ohio 44135
}

\section{SUMMARY}

Through the use of equivalent variable analogies, the authors demonstrate how an electrical subsystem can be modeled by an equivalent structural subsystem. This allows the electrical subsystem to be probabilistically analyzed by using available structural reliability computer codes such as NESSUS. With the ability to analyze the electrical subsystem probabilistically, we can evaluate the reliability of systems that include both structural and electrical subsystems. Common examples of such systems are a structural subsystem integrated with a healthmonitoring subsystem, and smart structures. Since these systems have electrical subsystems that directly affect the operation of the overall system, probabilistically analyzing them could lead to improved reliability and reduced costs. The direct effect of the electrical subsystem on the structural subsystem is of secondary order and is not considered in the scope of this work.

\section{INTRODUCTION}

Complex systems are often composed of subsystems of more than one engineering discipline (e.g., electrical, mechanical, chemical, nuclear, fluid, and structural). Although each of the subsystems can affect the total system and often affect other subsystems, the components that comprise the subsystems may vary. For example, a structural subsystem has material property, load, and dimension components, whereas a simple electrical subsystem has resistance, voltage, and current components. Each component in each of these subsystems varies in magnitude and/or direction. Because of the variability of the components and the various levels of sensitivity of their coupling, the complete system varies in its operations. In order to analyze the variability of such a complex system, the subsystems must be evaluated probabilistically (stochastically). And probabilistic evaluation of multidisciplinary systems requires computer codes that can analyze structural, thermal, chemical, fluid, and electrical subsystems. However, it is through such an evaluation that critical subsystems and components can be identified; this, in turn, can lead to reduced cost and improved reliability of the complex systems.

One probabilistic code used to evaluate structural subsystems is NESSUS (Numerical Evaluation of Stochastic Structures Under Stress); it has been developed for the NASA Lewis Research Center by Southwest Research Institute (refs. 1 and 2). This code can be used to evaluate structural subsystems with variable material properties, dimensions, and loadings. Possible modes of failure can be combined through fault tree analysis to determine the reliability (probability of failure) of the subsystem. Risk analysis that includes the subsystems' costs can also be performed with the code. However, this probabilistic structural code is limited to the analysis of structural and thermal-structural subsystems.

The work reported herein draws analogies between the variables of electrical and structural subsystems and then uses them to analyze several simple electrical circuits. These equivalent variables allowed an electrical 
subsystem to be probabilistically analyzed with the NESSUS computer code. This ability to analyze the subsystem probabilistically allowed us to evaluate the reliability of systems having both structural and electrical subsystems. In the future, identification of the critical components, whether structural or electrical, will make possible more reliable systems at reduced costs.

Common examples of such systems are a structural subsystem integrated with a health-monitoring subsystem (ref. 3), and smart structures (ref. 4). These systems have electrical subsystems that directly affect the operation of the overall system. For example, in a health-monitoring subsystem, electrical sensors inform system operators to make proper adjustments. If the monitoring system fails, the structural subsystem can be damaged or it can fail. Similarly, in smart structures that have an electrical subsystem in the form of piezoelectrical sensors and/or actuators (ref. 5), failure of the piezoelectric system can also result in failure of or damage to the structural subsystem.

The primary objective of this work was to determine the feasibility of using a structural code to probabilistically analyze electrical subsystems. Investigating the direct effect of the electrical subsystem on the structural subsystem, however, was beyond the scope of this work.

Analogies between other disciplines and the structures discipline can be demonstrated so that such subsystems can be probabilistically analyzed with a single code.

\section{ELECTRICAL AND STRUCTURAL ANALOGIES}

There are analogies between the variables in the equations used by various engineering disciplines; thus, similar equations can be used amongst the disciplines. Analog computers were the prime example of the use of such analogies (ref. 6). Here, in an inverse example, dc and ac circuits are analyzed by using structural analogies and computer codes developed to analyze structural systems.

A dc circuit can be analyzed by the following equation:

$$
I=\frac{V}{R}
$$

where $I$ is the current, $V$ the voltage, and $R$ the resistance. The equivalent equation for structural subsystems is

$$
F=k u
$$

where $F$ is the force, $k$ the stiffness of the structure, and $u$ the displacement. Equations (1) and (2) show that force $F$ is analogous to current $I$; displacement $u$ is analogous to voltage $V$; and flexibility $1 / k$ is analogous to resistance $R$. Though other combinations of analogies can be derived for the variables in these equations, those just presented result in consistent analogies, which will be used here. In addition, these analogies have a physical correlation in that force flows in a structure just as current flows in a circuit; the portion of force transferred to members depends on their flexibility just as the portion of current transferred to various paths depends on their resistance. And displacement is a function of force and flexibility just as a voltage change is a function of current and resistance of the circuit. Table I summarizes the variables that are equivalent in the simple electrical and the static structural subsystems. The makeup of these electrical circuits is limited to voltage and current sources and resistors. 
For electrical subsystems that include inductors and capacitors, a dynamic structural subsystem (ref. 7) can be used to model the electrical subsystem. The relationship for an inductor is

$$
I=\frac{1}{L} \int V d t
$$

where $L$ is the inductance, and $t$ is time. The similar equation for the structural model is

$$
F_{s}=k u=k \int \dot{u} d t
$$

where $\dot{u}$ is the velocity and $F$, is the stiffness force. Comparison of equations (3) and (4) shows flexibility is equivalent to inductance $L$, and velocity $\ddot{u}$ is analogous to voltage $V$.

The equation for a capacitor is

$$
I=C \frac{d V}{d t}
$$

where $C$ is the capacitance. The analogous equation for a structure is

$$
F_{l}=-m \ddot{i}
$$

where $F_{I}$ is the inertial force, and $\ddot{u}$ is the acceleration. In equations (5) and (6), mass $m$ is analogous to capacitance $C$.

The structural equation that is equivalent to the resistor equation in such a circuit is

$$
F_{D}=c \dot{i}
$$

where $F_{D}$ is the damping force, and $c$ is the damping. From equations (1) and (7) we can see that $c$ is analogous to the inverse of the resistance.

The linear equation to be solved in the electrical circuit is

$$
C \frac{d V}{d t}+\frac{V}{R}+\frac{1}{L} \int V d t=I
$$

which is analogous to the structural dynamics equation

$$
m \ddot{u}+c \dot{u}+k u=F(t)
$$

where $F$ is the forcing function. The equivalent variables for the ac circuit are summarized in table II. 
In both of the foregoing analogies, the force is taken to be analogous to the current. However, force is a vector and current is a scalar. Therefore, uniaxial structural models were used to eliminate the difficulties associated with the direction of forces when an electrical circuit was modeled. It is possible to use planar structures or nonplanar structures to model more complex electrical circuits. When nonplanar structures are used, care must be exercised to properly represent the analogies by the structural member's spatial position. The use of nonplanar electrical circuits, however, introduces comparable complexities in the analogy process.

\section{Direct Current Circuit Analyses}

The simple electrical circuit shown in figure 1(a) was probabilistically analyzed with the NESSUS computer code. The circuit was modeled as an equivalent structural subsystem by using the analogies previously presented. Figure 1(b) shows the structural model with node and element (encircled) numbers. Elements 1, 4, and 5 had nondimensional flexibilities of $0.001,0.004$, and 0.005 , respectively. These flexibilities correspond to the resistors of the circuit. Elements 2 and 3 were made 1000 times stiffer than element 1 to model a rigid member and allow the proper flow of forces (current) to members 4 and 5 . All elements were beam elements. Therefore, only axial degrees of freedom were allowed in order to prevent bending. Node sets $(2,3),(4,6)$, and $(5,7)$ are master and slave node sets for the different member sections connected at these locations. The model was subjected to a normalized displacement of 12 at nodes 8 and 9 to simulate the voltage source.

Several analyses were performed with the model. The stiffness of the elements (equivalent resistance) was varied by perturbing the cross-sectional height and the modulus of elasticity of the elements. Although either of these two variables could have been perturbed to model the variability in the stiffness (resistance), both were perturbed to determine their combined effect on the results. The assumed distributions for the perturbed variables are shown in table III along with assumed means and scatter expressed in percent of the mean. The scatter of the variables signifies expected fluctuations of the variable.

The cumulative probabilities of occurrence for the currents and for the voltage drop $\mathrm{V}_{1}$ are shown in figures 2 and 3. The 95th percentiles show values of 3763,2115 , and $1693 \mathrm{~A}$ for currents $\mathrm{I}_{1} \mathrm{I}_{2}$, and $\mathrm{I}_{3}$, respectively, and $3.79 \mathrm{~V}$ for voltage $\mathrm{V}_{1}$. These are values that have only a 5-percent chance of being exceeded. Deterministic analysis resulted in values of 3724,1655 , and $2069 \mathrm{~A}$ and $3.72 \mathrm{~V}$ for currents $I_{1}, I_{2}, I_{3}$, and voltage $V_{1}$, respectively. The deterministic values are approximately at the 50th percentile level for the cumulative distribution functions. From this comparison, we observe that slight differences will occur if the design of the circuit is based on a deterministic analysis rather than on the 95th percentile of a probabilistic analysis. However, a deterministic analysis does not provide the designer with information on the probabilities that the responses will be exceeded, and hence, on the reliability of the design.

Sensitivities are a measure of the effect that each variable has on the probability of the response. Averages of the sensitivities for the perturbed variables are depicted in figures 4 and 5 . The averages were used because the sensitivities varied little at various levels of probability.

The cross-sectional height and modulus of elasticity of the structural elements correspond to the flexibility, which is analogous to the resistance within the circuit. This analogy allows the notation (see figs. 4 and 5) for referring to the resistor perturbation and to the corresponding structural perturbations. For example, the sensitivity for resistance 1 (R1) caused by perturbing the analogous element's cross-sectional height is resistance 1-h (R1-h). Similarly, the R1 sensitivity induced by perturbing the modulus of elasticity of the element is represented by resistance $1-\mathrm{m}(\mathrm{R} 1-\mathrm{m})$. Therefore, both resistance $1-\mathrm{h}$ and resistance $1-\mathrm{m}$ should be considered in drawing conclusions about the sensitivity in the analysis caused by resistance 1 . 
Figure 4 shows that in the analysis for $I_{1}$ the sensitivities of the variables related to $R 1$ have a considerable effect. The height variable analogs of resistors 2 and 3 also have a significant effect on $I_{1}$, but the modulus of elasticity analogs do not. It can also be concluded from this figure that $R 3$ has a greater effect on the $I_{1}$ analysis that $R 2$ does. In the analysis for $I_{2}$, the sensitivities of the variables show that $R 2$ has the greatest effect on $I_{2}$, followed by R1. As would be expected, R3 has little effect on $\mathrm{I}_{2}$. Sensitivities of the variables in the analysis for $\mathrm{I}_{3}$ show just the opposite results, with $\mathrm{R} 3$ having the greatest effect, $\mathrm{R} 1$ the next greatest, and $\mathrm{R} 2$ the least. In the analysis of $V_{1}$, sensitivities show results similar to that of $I_{1}$, but with even more of an effect coming from R1.

The other voltages can be determined by examining the relative displacements (between nodes 7 and 9 for element 4, and nodes 6 and 8 for element 5) as opposed to absolute displacements. Although determining these voltages was not within the scope of this study, there is no fundamental limitation of the codes that would prevent such a determination.

A separate analysis was done to determine the probability of the voltage $V_{1}$ exceeding an allowable voltage (equivalent displacement) that was assumed to have a scatter range described by the distribution and parameters shown in table III. This resulted in a probability of failure of 0.18482 . If the allowable voltage were considered deterministic, with a value of $3.9028 \mathrm{~V}$, the probability of failure would drop to 0.0000019 . This is shown in figure 3 by the high probability that the resultant voltage would be less than the allowable voltage. Thus, including the scatter of the allowable significantly affects the analysis.

A circuit with a current source was also probabilistically analyzed. It and the equivalent structural model are shown in figure 6. Elements $1,2,5$, and 6 were 500 times stiffer than the next stiffest element, 8. Again, this was done to make these elements as rigid as possible, thereby allowing proper transfer of the forces (currents). Elements 3 and 4 correspond to $10-\Omega$ resistors, whereas elements 7 and 8 correspond to $20-$ and $5-\Omega$ resistors, respectively. A concentrated nondimensional load of 36 was placed at node 1 to simulate the current source. All nodes were allowed only horizontal translation except nodes 7,12 , and 14 , which were fixed. These boundary conditions removed bending effects to accurately model the circuit.

Several analyses were performed on this model. Table IV shows the distributional information about the perturbed variables used in the analyses. The cumulative probabilities of occurrence for currents $I_{1}, I_{2}, I_{3}$, and $I_{4}$ are shown in figure 7 , and the average sensitivity factors are expressed in figure 8 . The 95 th percentile values for currents $I_{1}, I_{2}, I_{3}$, and $I_{4}$ were $24.47,17.47,13.98$, and $3.51 \mathrm{~A}$, respectively. Deterministic analyses resulted in corresponding values of $21,15,12$, and 3 . The difference between the probabilistic and deterministic analyses is approximately 16.5 percent, but the deterministic analyses provide no information on the chance that the responses will be exceeded.

The sensitivities of the currents in the analyses showed that the current source had a very significant effect on all analyses of the currents. The resistors in which the current was found had the next largest effect. Other variables had an insignificant effect.

The cumulative probabilities of occurrence of voltages $\mathrm{V}_{1}, \mathrm{~V}_{3}$, and $\mathrm{V}_{4}$ are shown in figure 9 , and average sensitivity factors are shown in figure 10 . Voltage $V_{2}$ was not determined for reasons previously discussed. The curves for $V_{3}$ and $V_{4}$ overlap and are nearly identical, as would be expected. The 95 th percentile voltages for $\mathrm{V}_{1}, \mathrm{~V}_{3}$, and $\mathrm{V}_{4}$ were $245.25,69.83$, and 70.08 , respectively. Deterministic analyses resulted in values of 210,60 , and 60 for the same voltages. This is approximately a 16.8-percent difference between the probabilistic and deterministic analyses.

In this analysis, the sensitivities of the voltages showed that the current source had an even more significant effect than it did in the analyses of the currents. The sensitivities of all the resistors had an almost negligible effect. 


\section{Alternating Current Circuit Analysis}

A simple circuit (fig. 11(a)) containing a resistor, inductor, and capacitor was probabilistically analyzed. This was done by modeling the circuit as the structural system shown in figure 11(b) and using the NESSUS computer code. Because of the presence of a capacitor and inductor, a harmonic analysis was performed by using

the analogies listed in table II. A harmonic forcing function with an amplitude $(A)$ of 0.667 , a phase angle $(\phi)$ of $\pi / 2 \mathrm{rad}$, and a frequency $(\omega)$ of $1.0 \mathrm{rad}$ was placed at node 1 to simulate the current source. The governing equation for this problem is

$$
m u+c u+k u=A e^{-i(\omega t+\phi)}
$$

By choosing these forcing parameters, we modeled the imaginary current source shown in figure 11(a). Two beam elements were used to improve the solution even though only one element is required to model all three of the electrical components. To prevent any bending effects, only horizontal translation of nodes 1 and 2 was allowed. A lumped mass of 0.0001 was placed at node 1 to model the capacitor. This required that the mass density of the elements be very small so that the results would not be adversely affected. The total axial stiffness of the structure was set at 0.0333 to model the inductor. A damping factor $(\zeta)$ of 0.43651 was used; it relates to the resistor through equation (7) as follows:

$$
\zeta=\frac{c}{2 \sqrt{k m}}
$$

where $c$ is the damping (1/resistance), $k$ the stiffness, and $m$ the mass. The variables that were perturbed in the model are shown in table $\mathrm{V}$ with their corresponding distribution information. Inductance- 1 is analogous to perturbing the length of the structural members, which is its flexibility and is analogous to inductance.

The structure was analyzed twice. The first analysis determined the cumulative probability of occurrence for the imaginary component of the voltage (velocity). The second analysis determined the probability that the real component of the voltage would occur. The cumulative probability of occurrence results and the results of the sensitivity analyses are shown in figures 12 to 14 . Probabilistic analyses resulted in 95th percentile voltages of 1.02 and 20.90 for the imaginary and real components of the voltage, whereas deterministic analyses resulted in values of 0.96034 and 20.024 , respectively. There is approximately a 5-percent difference between the values obtained with the two types of analysis.

The sensitivities show that the variables related to inductance had the greatest effect on both analyses, and the current source had the next greatest effect. The resistance affected the analysis for the imaginary component of the velocity slightly, but had no effect on the analysis for the real component.

\section{POTENTIAL ENHANCEMENTS}

Enhancements to the structural computer code and/or modeling procedure would allow detailed analyses of complex electrical circuits. In this investigation, an effort was made to identify possible enhancements without incorporating them in the code. Some are described here.

Modifying the code to allow boundary conditions to be perturbed would permit analysis of the effect of a stochastic voltage source that would be modeled with a prescribed boundary displacement. Using springs as supports that can be perturbed might be a modeling remedy. However, the correct spring stiffness is not obvious. 
Models for overdamped electrical circuits (i.e., damping factor $>1$ ) are not accounted for by comparable structural analogs, and appropriate enhancements to the structural code will have to be made in order to model overdamped electrical circuits.

An enhancement that permits the analog for conductance, with an additional member that has no stiffness but has a density, is needed.

The direct input of the damping matrix may be a solution to analogous structures that do not have a constant damping ratio. This would apply to structural analogs for modeling parallel circuits (fig. 11).

Slight modifications to the code to allow the inertia, damping, and stiffness forces to be valid response outputs would also permit probabilistic analyses of the currents within the conductor, resistor, and inductor.

These enhancements would make it possible to probabilistically analyze structural systems that contain electrical systems such as health-monitoring systems and smart structures. Additional enhancements could be made to include the coupling effect between the electrical and structural systems. This would improve probabilistic system analyses and would lead to improved designs, from a safety as well as an economic viewpoint.

\section{CONCLUDING REMARKS}

We have shown that an electrical circuit can be probabilistically analyzed by using equivalent variables in the structural analysis code NESSUS. Therefore, system reliability analyses can be performed on systems containing both structural and electrical subsystems. There are other analogies that exist between engineering disciplines that should allow other types of subsystems to be probabilistically analyzed with the same code. The end result would be system reliability analyses of a complete system, including such subsystems as electrical, thermal, hydraulic, structural, and the like. Analyses of coupling between subsystems could eventually be included, thereby leading to realistic modeling of the assembled system. Such improved system analysis would lead to increased reliability and reduced cost of the system.

\section{ACKNOWLEDGMENT}

This work was made possible through the NASA/ASEE Summer Faculty Fellowship Program, for which the authors are grateful.

\section{REFERENCES}

1. Chamis, C.C.: Probabilistic Structural Analysis Methods for Space Propulsion System Components. NASA TM-88861, June 1986.

2. NESSUS Version 6.0 Release Notes, NASA Contract NAS3-24389, SwRI Project 06-3285, Southwest Research Institute and Vanderbilt University, June 1992.

3. Helmicki, A.; Kuo, F.; and Valley, D.: Rocket Engine Health Monitoring and Control: Some Connections and Their Implications. The Third Annual Health Monitoring Conference for Space Propulsion Systems, University of Cincinnati, 1991, pp. 323-347. 
4. Shah, D.K.; Chan, W.S.; and Joshi, S.P.: Finite Element Analysis of Plates with Piezoelectric Layers. AIAAV ASME/ASCE/AHS/ASC Structures, Structural Dynamics, and Materials Conference, 34th and Adaptive Structures Forum, Pt. 6, AIAA, Washington, DC, 1993, pp. 3189-3197.

5. Newnham, R.E.: Piezoelectric Sensors and Actuators: Smart Materials. Proceedings of the Annual Frequency Control Symposium, IEEE, Piscataway, NJ, 1992, pp. 513-524.

6. MacNeal, R.: Electrical Circuit Analogies for Elastic Structures, John Wiley \& Sons, Inc., New York, 1962.

7. Craig, R.: Structural Dynamics, John Wiley \& Sons, Inc., New York, 1981.

TABLE I.-ANALOGOUS VARI-

ABLES IN DIRECT CURRENT

CIRCUITS AND STATIC

STRUCTURES

\begin{tabular}{|l|l|}
\hline $\begin{array}{c}\text { Electrical } \\
\text { variables }\end{array}$ & \multicolumn{1}{c|}{$\begin{array}{c}\text { Equivalent } \\
\text { structural variables }\end{array}$} \\
\hline $\begin{array}{l}\text { Current, } I \\
\text { Voltage, } V \\
\text { Resistance, } R\end{array}$ & $\begin{array}{l}\text { Force, } F \\
\text { Displacement, } u \\
\text { Flexibility, } 1 / k\end{array}$ \\
\hline
\end{tabular}

TABLE II.-ANALOGOUS VARIABLES

IN ALTERNATING CURRENT

CIRCUITS AND DYNAMIC

STRUCTURES

\begin{tabular}{|l|l|}
\hline $\begin{array}{c}\text { Electrical } \\
\text { variables }\end{array}$ & $\begin{array}{c}\text { Equivalent structural } \\
\text { variables }\end{array}$ \\
\hline Current, $I$ & Force, $F$ \\
Voltage, $V$ & Velocity, $u$ \\
Resistance, $R$ & 1/Damping, $1 / c$ \\
Capacitance, $C$ & Mass, $m$ \\
Inductance, $L$ & Flexibility, $1 / k$ \\
\hline
\end{tabular}

TABLE III.-RANDOM VARIABLE STATISTICS

FOR DIRECT CURRENT CIRCUIT 1

\begin{tabular}{|l|c|c|c|}
\hline Variable & Distribution & Mean & $\begin{array}{c}\text { Scatter, } \\
\text { percent of mean }\end{array}$ \\
\hline Resistance & & & \\
$1-\mathrm{h}$ & Normal & 0.5 & 10.0 \\
$2-\mathrm{h}$ & Normal & .5 & \\
$3-\mathrm{h}$ & Normal & .5 & \\
1-m & Lognormal & 2000.00 & \\
2-m & Lognormal & 400.00 & \\
3-m & Lognormal & 500.00 & \\
Voltage 1" & & 3.9028 & 10.0 \\
(allowable) & Normal & \\
aused in one analysis only.
\end{tabular}


TABLE IV.-RANDOM VARIABLE STATISTICS

FOR DIRECT CURRENT CIRCUIT 2

\begin{tabular}{|c|c|c|c|}
\hline Variable & Distribution & Mean & $\begin{array}{c}\text { Scatter, } \\
\text { percent of mean }\end{array}$ \\
\hline Current source & Normal & 36.0 & 10.0 \\
\hline Resistance & & & \\
\hline 1-h & Normal & 0.5 & 10.0 \\
\hline 2-h & & & \\
\hline 3-h & & & \\
\hline 4-h & $\downarrow$ & $\downarrow$ & \\
\hline $1-\mathrm{m}$ & Lognormal & .2 & \\
\hline $2-m$ & & .2 & \\
\hline $3-m$ & & .4 & \\
\hline 4-m & $\downarrow$ & .1 & $t$ \\
\hline
\end{tabular}

TABLE V.-RANDOM VARIABLE STATISTICS

FOR ALTERNATING CURRENT CIRCUIT 1

\begin{tabular}{|l|c|c|c|}
\hline \multicolumn{1}{|c|}{ Variable } & Distribution & Mean & $\begin{array}{c}\text { Scatter, } \\
\text { percent of mean }\end{array}$ \\
\hline Current source & Lognormal & 0.667 & 10.0 \\
Resistance & Lognormal & .4365 & 5.0 \\
Inductance-h & Normal & .5 & 10.0 \\
Inductance-1 & Lognormal & 2.0 & 1.0 \\
Inductance-m & Lognormal & .1333 & 10.0 \\
\hline
\end{tabular}




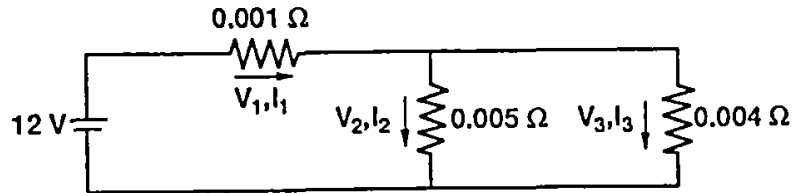

(a)

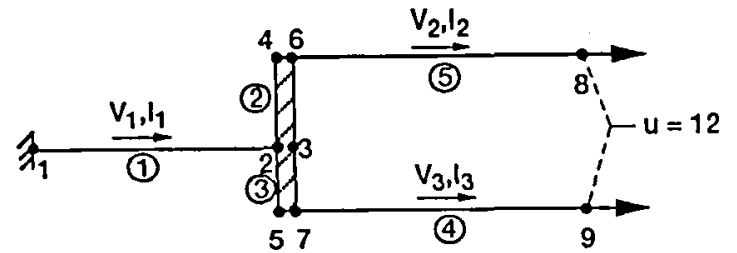

(b)

Figure 1. Direct current circuit 1 and analogous structural model. (a) Electrical do circuit 1. (b) Structural analog showing node and element (encircled) numbers.
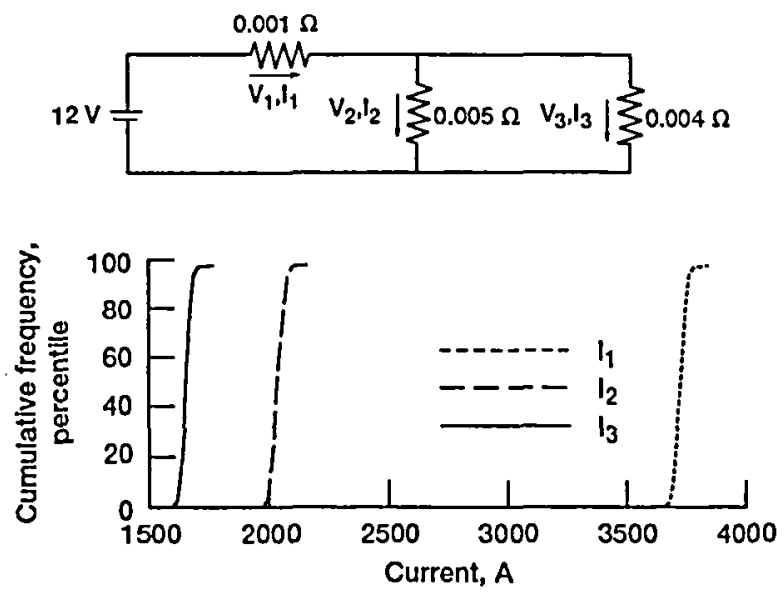

Figure 2. Probabilities of occurrence for currents of dc circuit 1. 

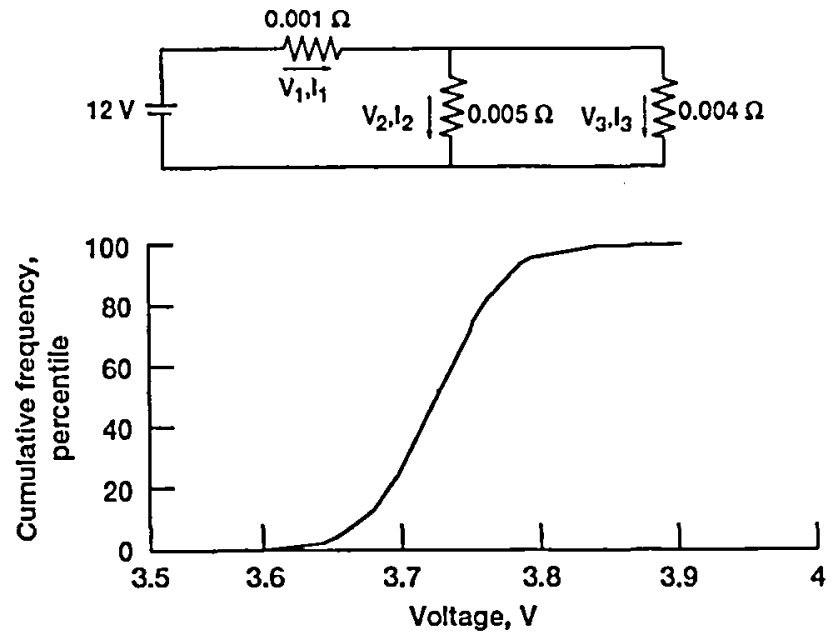

Figure 3. Probability of occurrence for $V_{1}$ of dc circuit 1.
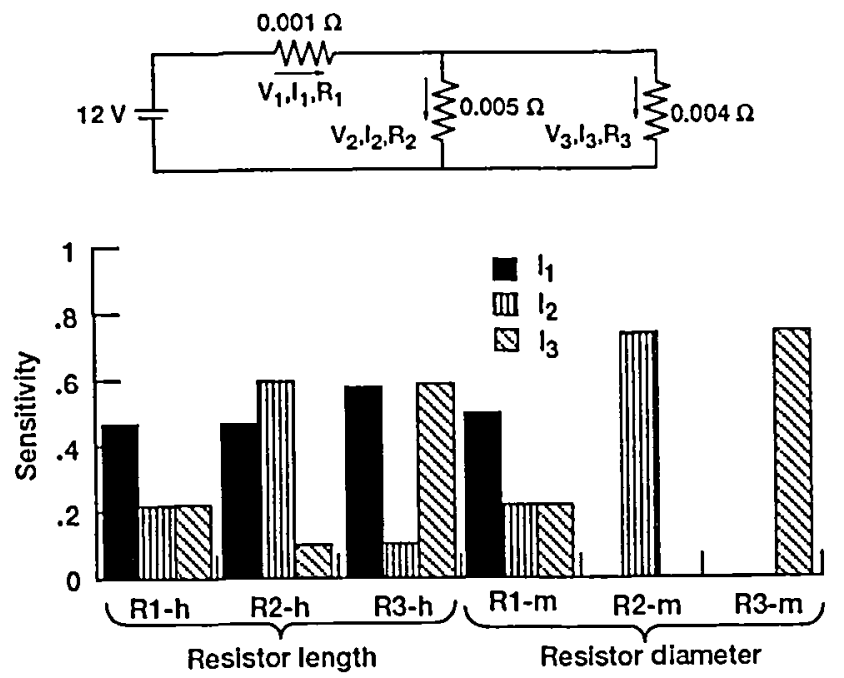

Figure 4. Sensitivities (relative units) for currents of dc circuit 1. 

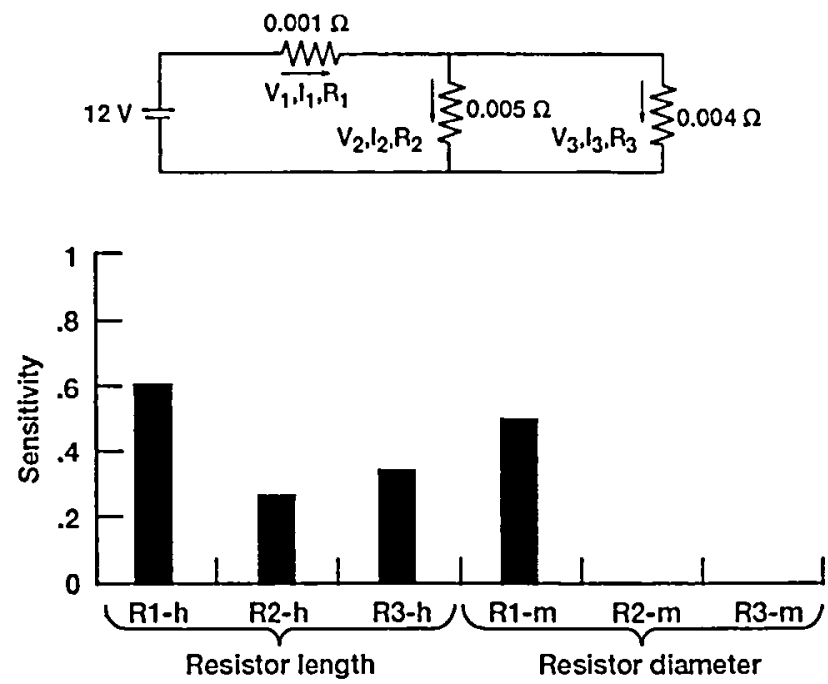

Figure 5. Sensitivities (relative units) for $V_{1}$ of dc circuit 1 .

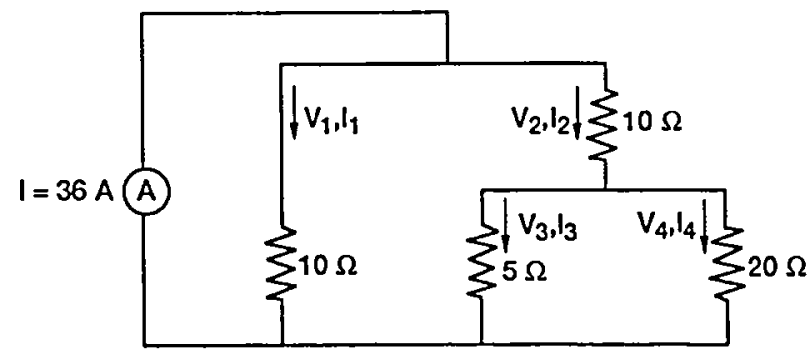

(a)

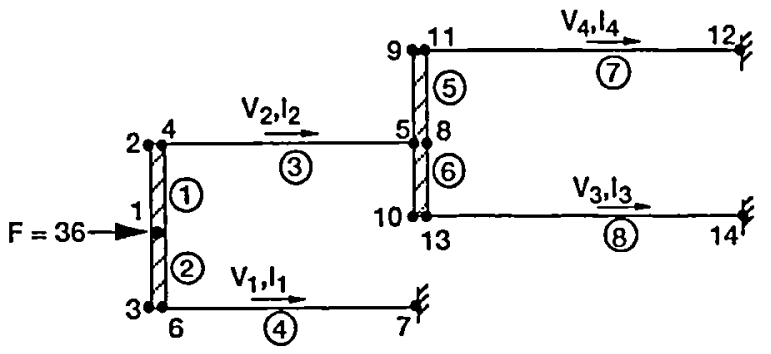

(b)

Figure 6. Direct current circuit 2 and analogous structural model. (a) Electrical dc circuit 2. (b) Structural analog showing node and element (encircled) numbers. 

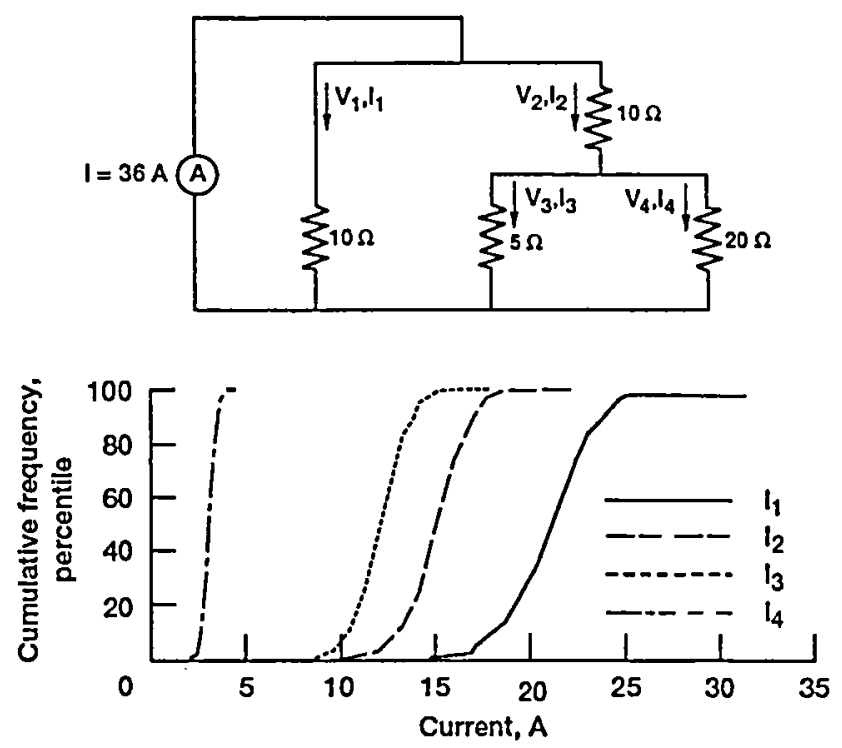

Figure 7. Probablities of occurrence for currents of dc circuit 2.
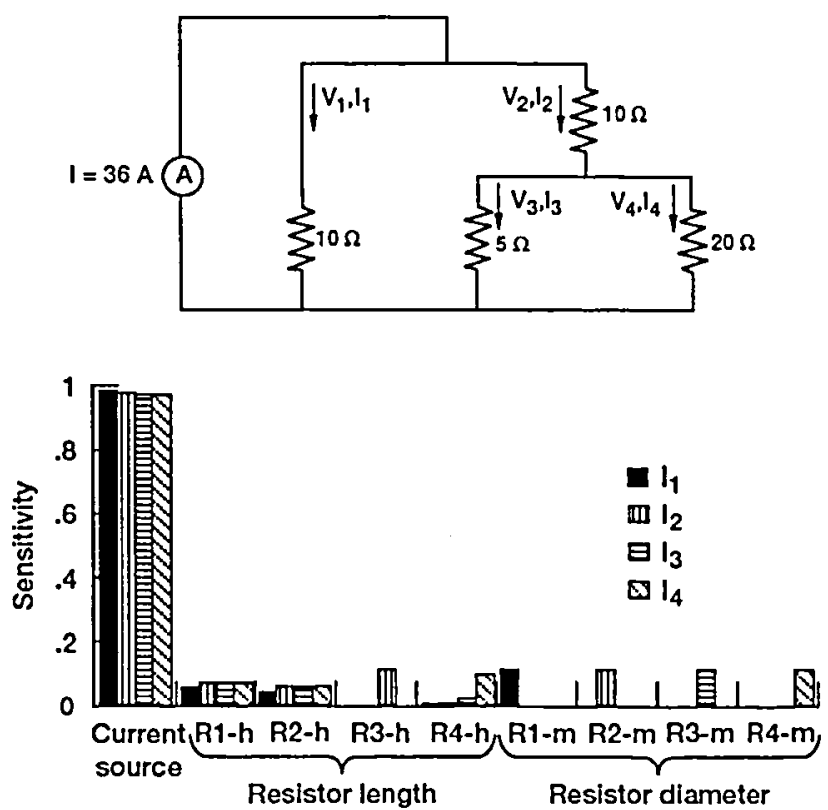

Figure 8. Sensitivities (relative units) for currents of dc circuit 2. 

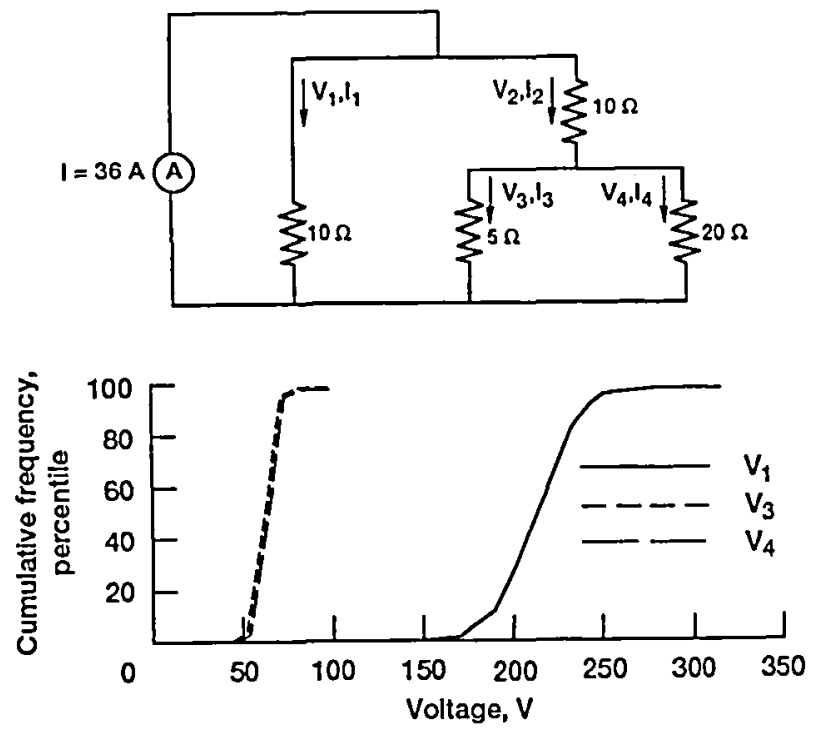

Figure 9. Probabilities of occurrence for voltages of dc circuit 2.
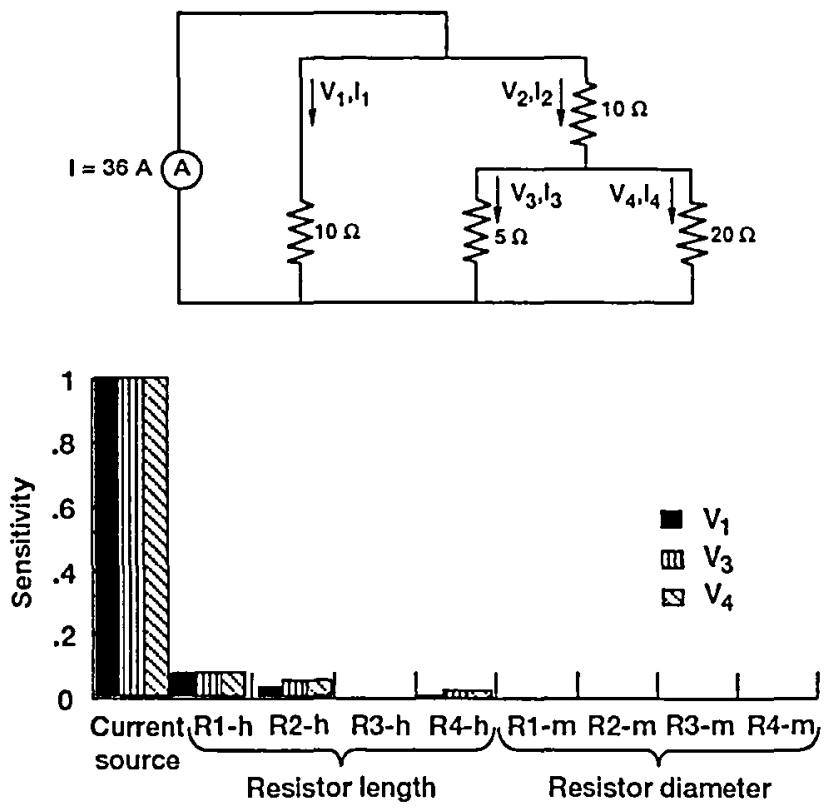

Figure 10. Sensitivities (relative units) for voltages of dc circuit 2. 


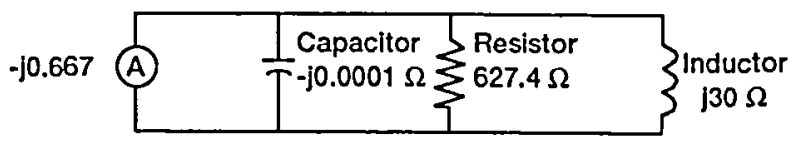

(a)

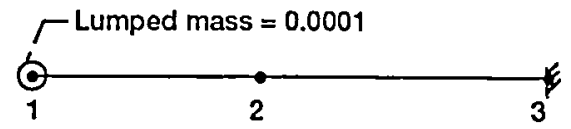

(b)

Figure 11. Alternating current circult and analogous structural model. (a) Electrical ac circuit. (b) Structural anolog.
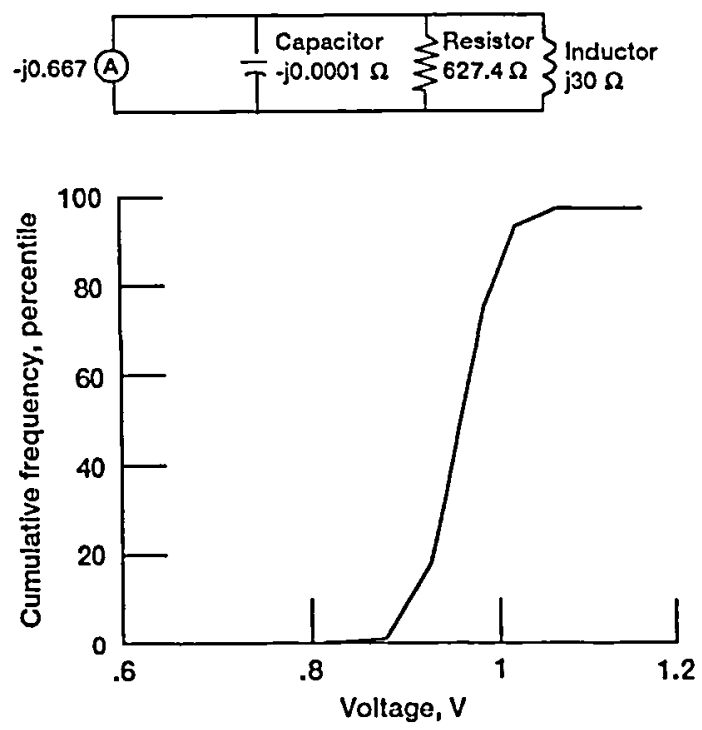

Figure 12. Probability of occurrence for imaginary voltage of ac circuit. 

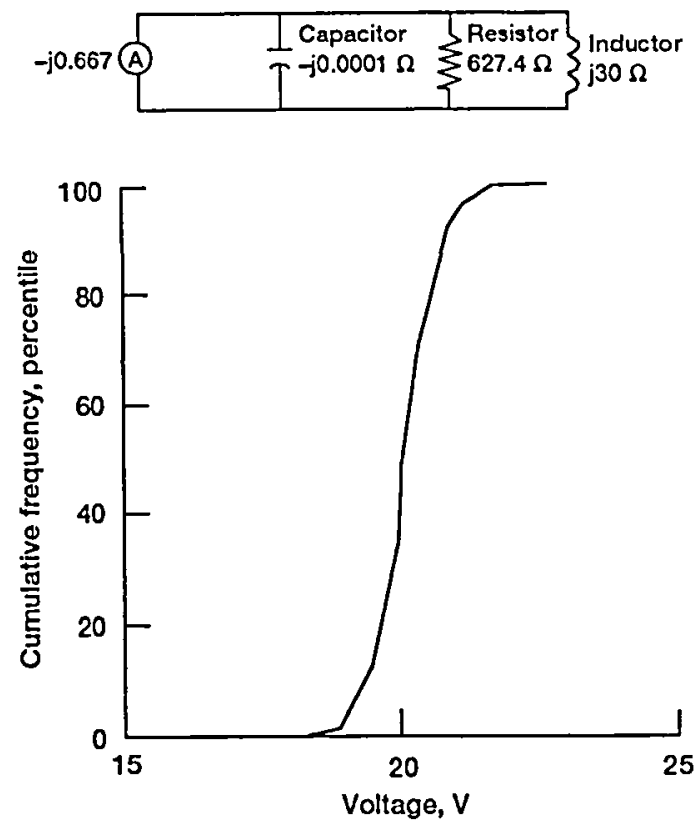

Figure 13. Probability of occurrence for real voltage of ac circuit.
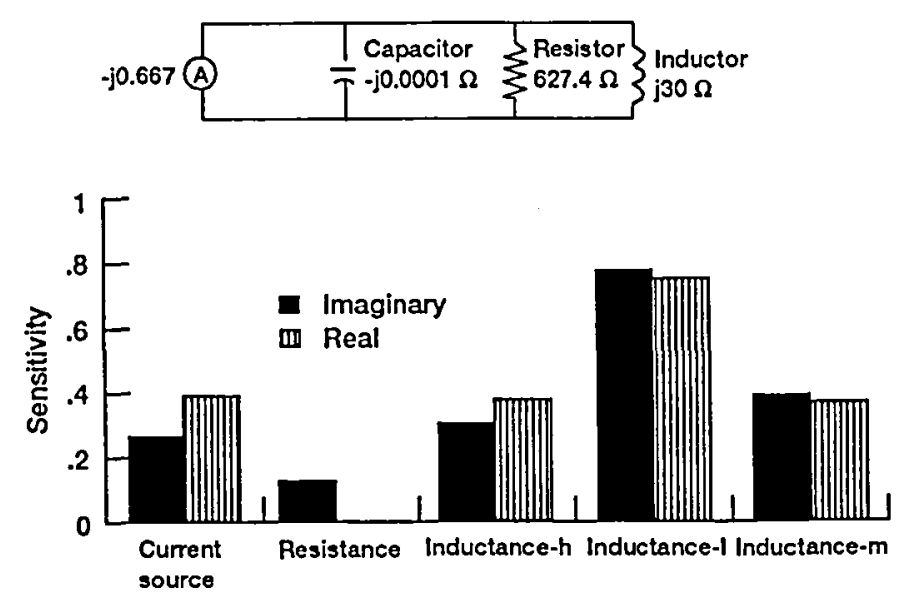

Figure 14. Sensitivitles (relative units) for voltages of ac circuit. 


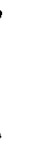


Public reporting burden for this collection of information is estimated to average 1 hour per response, including the time for reviewing instructions, searching existing data sources, gathering and maintaining the data needed, and completing and reviewing the collection of information. Send comments regarding this burden estimate or any other aspect of this collection of information, including suggestions for reducing this burden, to Washington Headquarters Services, Directorate for Information Operations and Reports, 1215 Jefferson Davis Highway, Suite 1204, Arlington, VA 22202-4302, and to the Office of Management and Budget, Paperwork Reduction Project (0704-0188), Washington, DC 20503.

\begin{tabular}{|l|l|l|}
\hline 1. AGENCY USE ONLY (Leave blank) & $\begin{array}{r}\text { 2. REPORT DATE } \\
\text { March } 1994\end{array}$ & $\begin{array}{r}\text { 3. REPORT TYPE AND DATES COVERED } \\
\text { Technical Memorandum }\end{array}$ \\
\hline
\end{tabular}

4. TITLE AND SUBTITLE

5. FUNDING NUMBERS

Structural Probability Concepts Adapted to Electrical Engineering

6. AUTHOR(S)

Eric P. Steinberg and Christos C. Chamis

\section{PERFORMING ORGANIZATION NAME(S) AND ADDRESS(ES)}

National Aeronautics and Space Administration

Lewis Research Center

Cleveland, Ohio 44135-3191

9. SPONSORING/MONITORING AGENCY NAME(S) AND ADDRESS(ES)

National Aeronautics and Space Administration

Washington, D.C. 20546-0001
WU-584-03-11

8. PERFORMING ORGANIZATION REPORT NUMBER

E-8493

10. SPONSORING/MONITORING AGENCY REPORT NUMBER

NASA TM-106499

AIAA-94-1383

\section{SUPPLEMENTARY NOTES}

Prepared for the 35th AIAA Structures, Structural Dynamics and Materials Conference sponsored by the American Institute of Aeronautics and Astronautics, Hilton Head, South Carolina, April 18-20, 1994. Eric P. Steinberg, Ohio University, Department of Civil Engineering, Stocker Center, Athens, Ohio 45701 and Christos C. Chamis, NASA Lewis Research Center. Responsible person, Christos C. Chamis, organization code 5200, (216) 433-3252.

12a. DISTRIBUTION/AVAILABILITY STATEMENT

12b. DISTRIBUTION CODE

Unclassified - Unlimited

Subject Category 39

\section{ABSTRACT (Maximum 200 words)}

Through the use of equivalent variable analogies, the authors demonstrate how an electrical subsystem can be modeled by an equivalent structural subsystem. This allows the electrical subsystem to be probabilistically analyzed by using available structural reliability computer codes such as NESSUS. With the ability to analyze the electrical subsystem probabilistically, we can evaluate the reliability of systems that include both structural and electrical subsystems. Common examples of such systems are a structural subsystem integrated with a health-monitoring subsystem, and smart structures. Since these systems have electrical subsystems that directly affect the operation of the overall system, probabilistically analyzing them could lead to improved reliability and reduced costs. The direct effect of the electrical subsystem on the structural subsystem is of secondary order and is not considered in the scope of this work.

\section{SUBJECT TERMS}

15. NUMBER OF PAGES

Analogies; Electrical; Structural; Probability; Sensitivity; System reliability

17. SECURITY CLASSIFICATION OF REPORT Unclassified

18. SECURITY CLASSIFICATION
OF THIS PAGE
Unclassified

19. SECURITY CLASSIFICATION OF ABSTRACT Unclassified 
National Aeronautics and

Space Administration

Lewis Research Center

21000 Brookpark Rd.

Cleveland, $\mathrm{OH}$ 44135-3191

OHiclal Business

Penally for Pilvate Use $\$ 300$

POSTMASTER: If Undellverable - Do Not Return

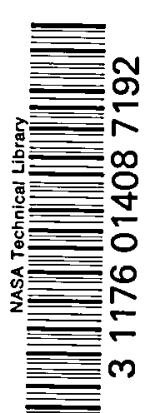

